\title{
High-Frequency AC Electrospray Ionization Source for Mass Spectrometry of Biomolecules
}

\author{
Nishant Chetwani, ${ }^{\text {a }}$ Catherine A. Cassou, ${ }^{\mathrm{b}}$ David B. Go, ${ }^{\mathrm{c}}$ and \\ Hsueh-Chia Chang ${ }^{\mathrm{a}}$ \\ a Department of Chemical and Biomolecular Engineering, University of Notre Dame, Notre Dame, Indiana, \\ USA \\ ${ }^{\mathrm{b}}$ Department of Chemistry and Biochemistry, University of Notre Dame, Notre Dame, Indiana, USA \\ ${ }^{\mathrm{c}}$ Department of Aerospace and Mechanical Engineering, University of Notre Dame, Notre Dame, Indiana, \\ USA
}

A novel high-frequency alternating current (AC) electrospray ionization (ESI) source has been developed for applications in mass spectrometry. The AC ESI source operates in a conical meniscus mode, analogous to the cone-jet mode of direct current (DC) electrosprays but with significant physical and mechanistic differences. In this stable conical-meniscus mode at frequencies greater than $50 \mathrm{kHz}$, the low mobility ions, which can either be cations or anions, are entrained within the liquid cone and ejected as droplets that eventually form molecular ions, thus making AC ESI a viable tool for both negative and positive mode mass spectrometry. The performance of the AC ESI source is qualitatively shown to be frequency-dependent and, for larger bio-molecules, the AC ESI source produced an ion signal intensity that is an order of magnitude higher than its DC counterpart. (J Am Soc Mass Spectrom 2010, 21, 1852-1856) (C) 2010 Published by Elsevier Inc. on behalf of American Society for Mass Spectrometry

$\mathrm{T}$ The emergence of new "soft" ionization techniques over the past two decades in the form of direct current (DC) electrospray ionization (ESI) [1] and matrix-assisted laser desorption ionization (MALDI) [2] in mass spectrometry (MS) has revolutionized the study of proteins and peptides and contributed significantly to biological and biomedical research. In contrast to ionization techniques such as fast atom bombardment (FAB), electron impact (EI), and thermal ionization that tend to fragment large molecules, ESI and MALDI minimize fragmentation, hence making them appropriate for analysis of biomolecules [3, 4], polymeric macromolecules [5], and the study of the environmental implications of hazardous materials [6]. The operating principle of ESI is that it generates charged droplets from the tip of a static conical liquid structure [7] known as Taylor's cone [8]. These charged droplets, containing analyte molecules present in the liquid, eventually form molecular ions by either multiple Raleigh fission events or, alternatively, via the charge residue mechanism [9]. In this study, we introduce another variant of ESI that is obtained by application of high-frequency alternating current (AC) potential to generate charged droplets for mass spectrometry.

In previous studies by the authors' group, it has been shown that in a certain window of frequency-voltage phase space, a steady, slender conical meniscus [10-12]

Address reprint requests to Dr. D. B. Go, Department of Aerospace and Mechanical Engineering, University of Notre Dame, Notre Dame, IN, 46556, USA. E-mail: dgo@nd.edu or Dr. H.-C. Chang, Department of Chemical and Biomolecular Engineering, University of Notre Dame, Notre Dame, IN, 46556, USA. E-mail: hchang@nd.edu is obtained using a high-frequency AC electrospray. As shown in Figure 1b, these AC cones, which have a half cone angle of $\sim 11^{\circ}$ and exhibit a continuous longitudinal growth in time are significantly different from static DC Taylor cones (with half cone angle $\sim 49^{\circ}$ ), pulsating electrosprays [13], or low-frequency $(<10 \mathrm{kHz}) \mathrm{AC}$ electrosprays [14] (Supplementary Material showing an $\mathrm{AC}$ cone of ethanol solvent at an applied AC potential of frequency $80 \mathrm{kHz}$ and magnitude $\sim 5.5 \mathrm{kV}$ (peak to peak) along with the preliminary sensitivity curve and positive mode mass spectra of cytochrome $c$, which can be found in the electronic version of this article). The formation of the $\mathrm{AC}$ cone is due to the difference in the mobility of liquid phase anions and cations that leads to a build up of the low mobility species in the cone because of insufficient time to relax onto the surface of the meniscus when the AC signal switches polarity-a condition induced when the inverse relaxation time scale is less than the high-frequency of AC signal [12]. This asymmetric effect leads to a net space charge in the cone from the accumulation of low-mobility ions, and the resulting Coulombic repulsion sustains the AC cone. This is again in direct contrast to DC electrosprays in which charge separation occurs and equilibrium is established instantaneously, leading to no net charge within the liquid cone, and all the space charge generated during ionization exists on the liquid jet that emanates from the tip of the cone.

In this work, the efficacy of high-frequency AC ESI as an ionization source for mass spectrometry (MS) is explored. The performance of the AC ESI source is compared with a DC ESI using representative analytes 

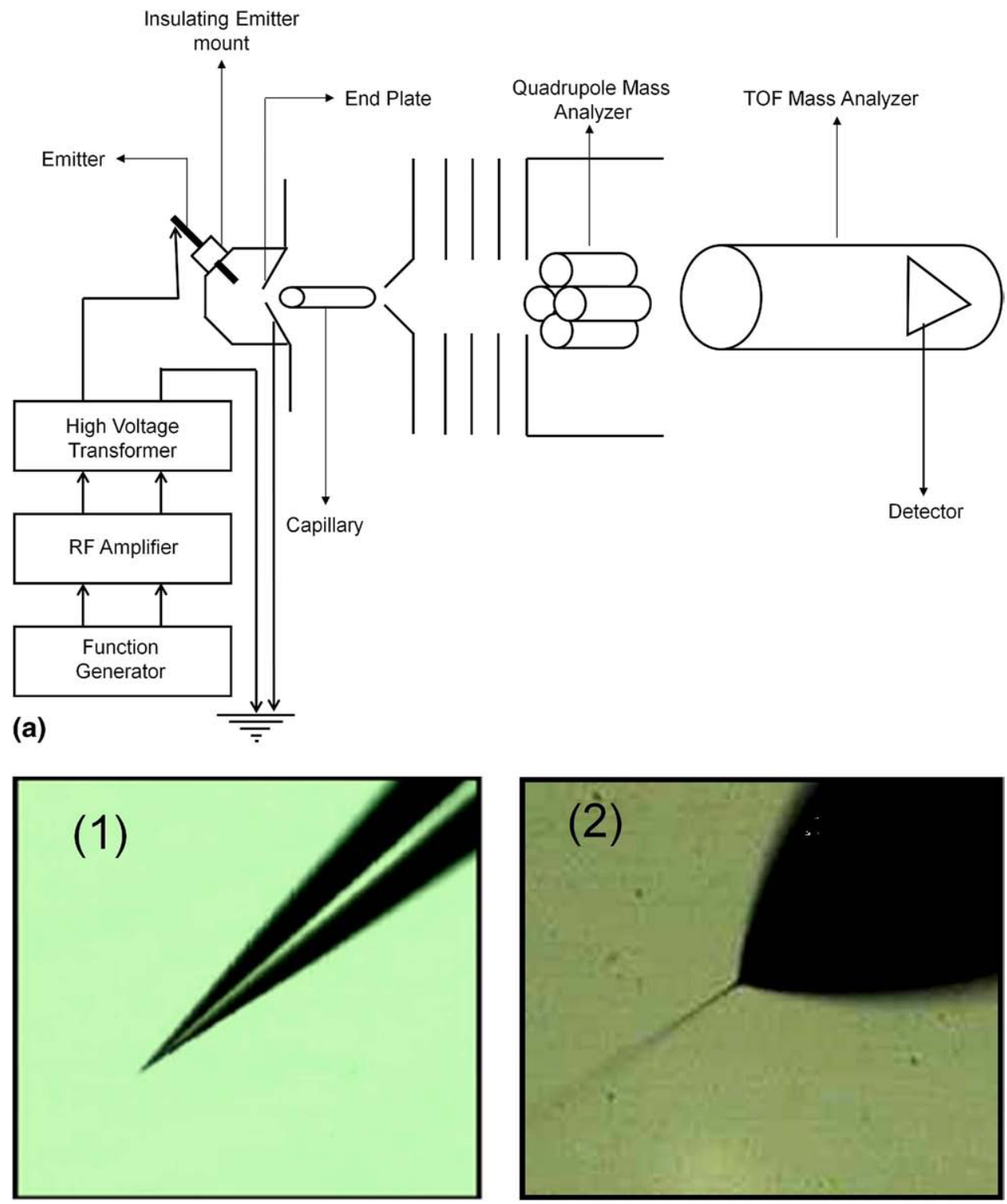

(b)

Figure 1. (a) Schematic of AC ESI MS experimental apparatus for AC ESI. (b) The image illustrating (1) an AC cone and (2) a DC cone of ethanol.

(10-mer oligonucleotides, cytochrome $c$, and myoglobin), and qualitative differences are discussed. Additionally, basic parametric characteristics of AC ESI are presented.

\section{Experimental}

High purity HPLC grade representative 10-mer oligonucleotides with a molecular mass $\mathrm{M} \sim 3040$ Da were obtained from Invitrogen Inc. and were prepared in 1:1 (vol/vol) acetonitrile (Sigma Aldrich, St. Louis, MO, USA) and deionized water. High purity grade oligonucleotide samples were used to ensure that the mass spectra obtained were clean and interference from impurities present in the sample was minimized. The protein samples, cytochrome $c$ with a molecular mass
$\mathrm{M} \sim 12,400 \mathrm{Da}$ (Sigma Aldrich) and myoglobin with molecular mass $\mathrm{M} \sim 17,000 \mathrm{Da}$ (Sigma Aldrich), were also prepared in 1:1 ratio (vol/vol) of acetonitrile and de-ionized water with an addition of 1:1000 formic acid to facilitate the formation of positive ions.

Mass spectra were collected on both an UltrOTOF-Q mass spectrometer (Bruker Daltonics Inc.) equipped with a hexapole in series with a quadrupole, and coupled with a time-of-flight (TOF) mass analyzer and an Esquire 3000+ (Bruker Daltonics Inc., Billerica, MA, USA) equipped with quadrupole mass analyzer, and both were equipped with a native DC ESI source and chamber. For AC ESI experiments, the end plate was set to $0 \mathrm{~V}$ and a high-frequency $\mathrm{AC}$ potential was directly applied to the emitter, as shown in Figure 1a. To avoid any damage to the 
equipment, the vendor's metal ESI chamber was customized, and a new emitter mount made out of insulating material was used in all the experiments. For the DC ESI experiments, two electrical configurations were used. In Configuration I, the end plate voltage was set to $3200 \mathrm{~V}$ using the inbuilt power source of the mass spectrometer while the emitter was kept at ground, which is the standard operation for these mass spectrometers. In Configuration II, for direct comparison with AC ESI, an external DC voltage source applied a high potential directly to the emitter while the end plate was set to $0 \mathrm{~V}$. This mimicked the electrical configuration of the AC ESI experiment. In both configurations, the DC ESI potential difference was set to equal the root mean square (RMS) voltage of the AC signal. The ion optics were set to optimize the signal intensity and remained constant between AC and DC ESI experiments for comparison. Additionally, in both AC and DC ESI experiments, nitrogen gas was used as a nebulizing gas at a pressure of 2 bars to aid droplet formation and stabilize the electrospray, and also as a counter-flow drying gas at a flow rate of $5 \mathrm{~L} / \mathrm{min}$ to enhance desolvation. A sample flow rate of $4 \mu \mathrm{L} / \mathrm{min}$ was used.
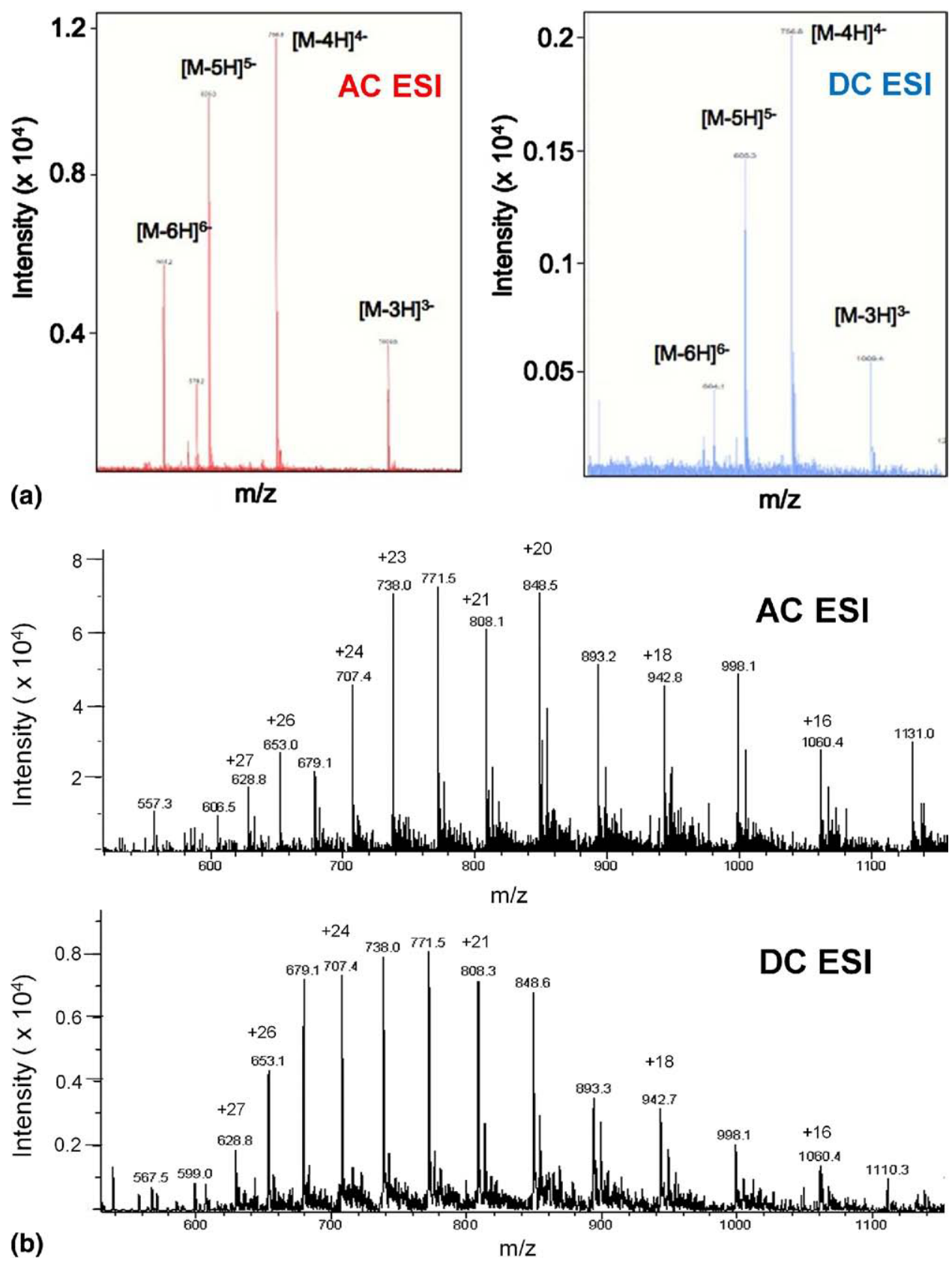

Figure 2. (a) Negative mode mass spectra of 10 base oligonucleotides $(75 \mu \mathrm{M})$ using (1) AC ESI (at $80 \mathrm{kHz}$ ) and (2) DC ESI in Configuration I. (b) Positive mode mass spectra of myoglobin $(40 \mu \mathrm{M})$ using (1) AC ESI (at $80 \mathrm{kHz}$ ) and (2) DC ESI in Configuration I. 


\section{Results and Discussion}

Figure 2a shows a side-by-side comparison of negative mode mass spectra obtained using high-frequency AC ESI and Configuration I DC ESI for $100 \mu \mathrm{M} 10$ base oligonucleotides. It is evident that the qualitative behavior of both ionization techniques is comparable in the sense that ions with same charge states $(\mathrm{m} / \mathrm{z})$ are produced. This observation indicates that the mechanism for the formation of ions in the gas phase, either by successive Rayleigh fission or desorption, is the same for both AC and DC ESI. The striking difference between the two mass spectra is in terms of the ion intensity, where the AC ESI signal is an order of magnitude more intense than the DC ESI signal, a result of two mechanisms in the formation of AC electros- prays as discussed later. A similar trend is depicted in Figure $2 \mathrm{~b}$ for a positive mode mass spectrum of $40 \mu \mathrm{M}$ myoglobin using Configuration I DC ESI experiments, and again AC ESI produced a nearly order of magnitude increase in the signal intensity. It should be noted that these spectra are illustrative of consistent trends that were observed with various samples, and that AC ESI spectra were obtained for concentrations a low as 2 $\mu \mathrm{M}$ with $\mathrm{S} / N>10$. DC ESI, in comparison, yielded much lower $\mathrm{S} / \mathrm{N}$ ratio at the same concentrations. It is believed with further optimization, even better AC ESI performance is possible.

The mobility of the oligonucleotide anions [M $\mathrm{nH}]^{n-}$ (or $[\mathrm{M}+\mathrm{nH}]^{n+}$ for myoglobin) is orders of magnitude lower than that of the other ions present in

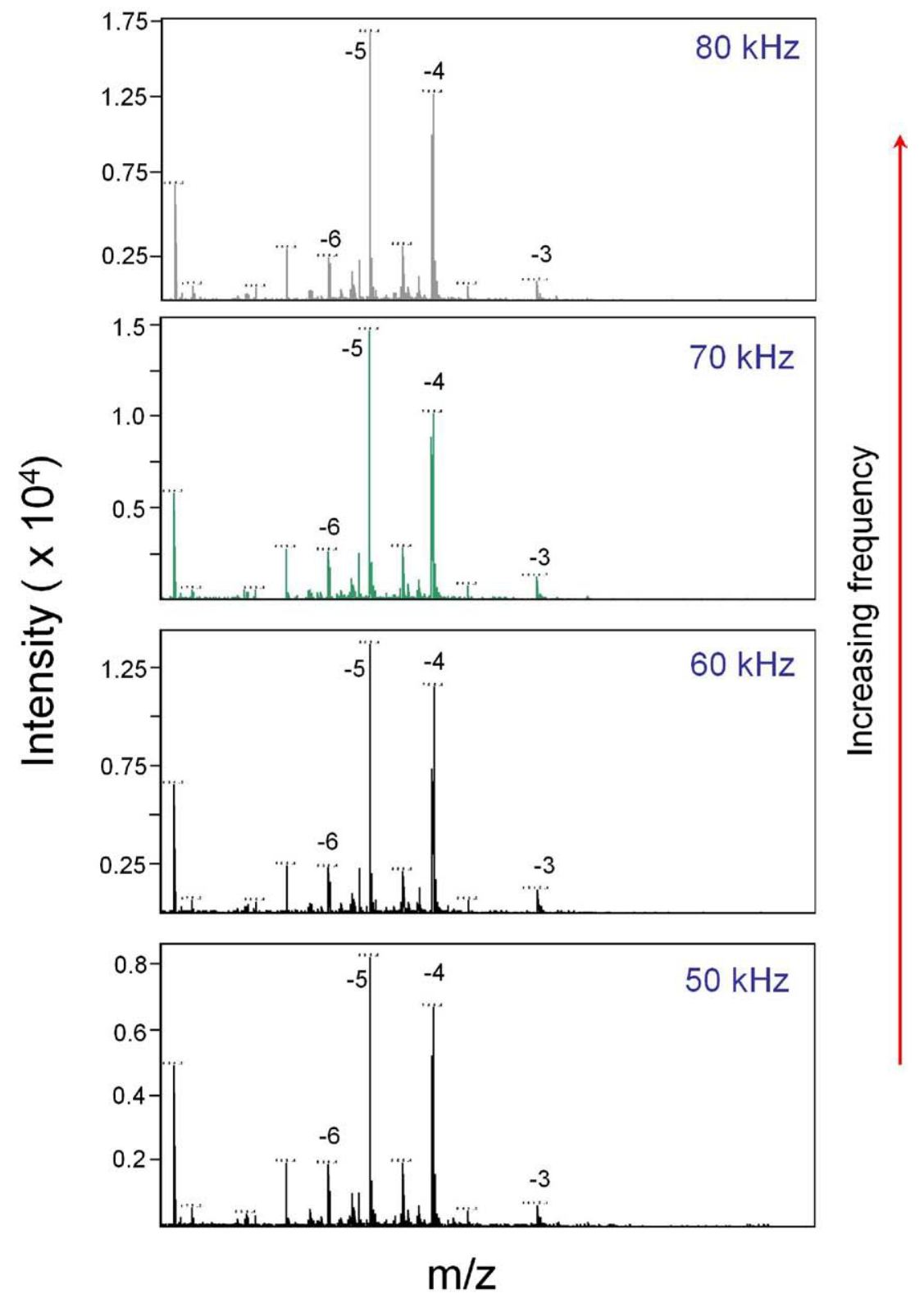

Figure 3. Negative mode AC ESI mass spectra of 10 base oligonucleotide $(100 \mu \mathrm{M})$ operated at frequencies of (a) $50 \mathrm{kHz}$, (b) $60 \mathrm{kHz},(\mathbf{c}) 70 \mathrm{kHz}$, and (d) $80 \mathrm{kHz}$. 
the solution, and they are preferentially entrained towards the tip of the $\mathrm{AC}$ cone, resulting in a higher "pseudo" concentration of charged biomolecule near the tip of the cone. Additionally, without electrons populating the ejected drops, a coarser size distribution of droplets ejected from the tip of the AC cone indicates that the surface charge density on a droplet is much less than that of droplets ejected from a DC cone. The smaller surface charge density delays Rayleigh fission and, due to the reduced electrostatic repulsion between the droplets, the plume of ejected droplets (and the subsequent generations of droplets obtained by Rayleigh fission) for AC electrosprays is much thinner in comparison to that of DC cones. This was confirmed by observing the AC and DC cone cases directly under an optical microscope in which the plume of droplets were clearly visible due to scattering of fluorescent light. As such, a more directed beam of ions enters the MS, minimizing ion loss. These two unique characteristics of AC cone, viz. preferential entrainment of low mobility ions in the cone and a more confined plume of ejected droplets, together contribute to the higher AC ESI signal intensity shown in Figure 2.

The pronounced effect of preferential entrainment of ions is evident from Figure 3, which depicts the mass spectra of representative oligonucleotides at different applied AC frequencies. As the frequency increases, a greater number of half $\mathrm{AC}$ cycles are accommodated over a given time. As such, at higher frequencies, the degree of ionization and subsequent concentration of oligonucleotides after every half AC cycle is enhanced within the $\mathrm{AC}$ cone resulting in higher signal intensities for higher frequencies (Figure 3). However, as shown by the modest increase from 70 to $80 \mathrm{kHz}$, it is expected that at some frequency the signal will be optimized, and this will be explored in a future study.

In contrast to the negative mode mass spectrum of oligonucleotides, AC ESI can also be used for positive mode MS (e.g., cytochrome $c$ and myoglobin). This is again due to the generation of protonated protein molecules in the AC cone that are driven toward the tip of the cone and eventually ejected from the cone, as shown in Figure $2 \mathrm{~b}$ for myoglobin and in the supplementary material for cytochrome $c$ (where DC ESI was operated in configuration II). As such, the high-frequency AC field can produce both negative and positive ions depending on the mobility of the species. When the low mobility ions are cations, AC can be used for positive mode mass spectrometry and vice versa for anions.

\section{Conclusions}

AC ESI has been demonstrated as a viable soft ionization method for mass spectrometry, with distinct advantages over DC ESI owing to the preferential entrainment mechanism. Moreover, the more confined and directed beam of drops (and hence ions) generated by AC ESI, in conjunction with pre-concentration of low mobility ions, lead to a better signal intensity poten- tially reducing the limit of detection by an order of magnitude. In addition to enhanced signal intensity, AC ESI can be used for in situ separation of undesirable high mobility ions (like $\mathrm{Na}^{+}$and $\mathrm{K}^{+}$) that are likely to interfere with mass spectra by forming adducts with target analyte molecules. The variation of the mass spectra as a function of frequency may lead to a bispectral characterization of heterogeneous samples, particularly if selective fragmentation can be induced for more fragile molecules by a negative ramp of the frequency. The potential union of AC ESI with nanospray emitters and use in series with HPLC could ultimately result in cleaner mass spectra and reduction in the limits of detection by orders of magnitude, making AC MS ESI mass spectrometry a promising tool for the analysis of samples with ultra low concentration.

\section{Acknowledgments}

N.C. and H.C.C. acknowledge financial support from NSFIDBR0852741 and the Center for Applied Mathematics at the University of Notre Dame, Notre Dame. C.A.C. acknowledges funding through a Clare Booth Luce Fellowship and Notre Dame Glynn Family Honors Program Grant. D.B.G. acknowledges funding from the University of Notre Dame Faculty Scholarship Award.

\section{Appendix A Supplementary Material}

Supplementary material associated with this article may be found in the online version at doi:10.1016/ j.jasms.2010.06.023.

\section{References}

1. Fenn, J. B.; Mann, M.; Meng, C. K.; Wong, S. F.; Whitehouse, C. M. Electrospray Ionization for Mass Spectrometry of Large Biomolecules. Science 1989, 246, 64-71.

2. Tanaka, K.; Waki, H.; Ido, Y.; Akita, S.; Yoshida, Y.; Yoshida, T. Protein and Polymer Analyses up to $m / z \quad 100,000$ by Laser Ionization Time of Flight Mass Spectrometry. Rapid Commun. Mass Spectrom. 1988, 2, 151-153.

3. Griffin, J. T.; Smith, L. M. Single-Nucleotide Polymorphism Analysis by MALDI-TOF Mass Spectrometry. TIBTECH 2000, 18, 77-84.

4. Miranker, A.; Robinson, C. V.; Radford, S. E.; Dobson, C.M. Investigation of Protein Folding by Mass Spectrometry. FASEBJ 1996, 10, 93-101.

5. Ladaviere, C.; Lacroix D. P.; Delolme, F. First Systematic MALDI/ESI Mass Spectrometry Comparison to Characterize Polystyrene Synthesized by Different Controlled Radical Polymerization. Macromolecules 2009, 42, 70-84.

6. Rostad, C. E.; Hostettler, F. D. Profiling Refined Hydrocarbon Fuels Using Polar Components. Environ. Forensics 2007, 8, 129-137.

7. Zeleny, J. Instability of Electrified Surfaces. Phys. Rev. 1917, 10, 1-6.

8. Taylor, G. I. Disintegration of Water Drops in an Electric Field. Proc. R. Soc. Lond. A 1964, 280, 383-397.

9. Kebarle, P. A Brief Overview of the Present Status of the Mechanisms Involved in Electrospray Mass Spectrometry. J. Mass Spectrom. 2000, 35, 804-817.

10. Maheshwari, S.; Chang, H.-C. Anomalous Conical Menisci Under an AC Field-Departure from DC Taylor Cone. App. Phys. Lett. 2006, 89(1/3), 234103.

11. Maheshwari, S.; Chang, H.-C. Effects of Bulk Charge and Momentum Relaxation Time Scales on AC Electrospraying. J. App. Phys. 2007, 102(1/6), 034902

12. Chetwani, N.; Maheshwari, S.; Chang, H.-C. Universal Cone Angle of AC Electrosprays Due to Net Charge Entrainment. Phys. Rev. Lett. 2008, 101(1/4), 204501.

13. Marginean, I.; Nemes, P.; Vertes, A. A Stable Regime in Electrosprays. Phys. Rev. E 2007, 76(1/6), 026320.

14. Wang, P.; Maheshwari, S.; Chang, H.-C. Polyhedra Formation and Transient Cone Ejection of a Resonant Microdrop Forced by an AC Electric Field. Phys. Rev. Lett. 2006, 96(1/4), 254502. 